\title{
Neue Therapieoption für ALK-positive Patienten
}

Für ALK-positive Patienten mit nicht kleinzelligem Bronchialkarzinom (NSCLC) wurde das Spektrum der personalisierten Therapie mit dem TyrosinkinaseInhibitor Ceritinib ${ }^{1}$ erweitert. Seit dem 1. Juli 2015 steht das Arzneimittel für mit Crizotinib vorbehandelte ALK-positive NSCLC-Patienten zur Verfügung.

Fusionsproteine der anaplastischen Lymphom-Kinase (ALK) werden bei ca. $5 \%$ der Patienten mit NSCLC identifiziert, mit einem höheren Anteil bei Jüngeren, Nichtrauchern und Adenokarzinom-Patienten. Bei diesen Patienten konnte bisher nur Crizotinib eingesetzt werden. Die Symptomremission mit dieser Substanz erfolgt sehr rasch und die Tumoren sind innerhalb von 6 Wochen im CT deutlich geschrumpft. Allerdings kommt es bereits im 1. Jahr der Crizotinib-Therapie durch das Auftreten von Resistenzen zu fortschreitendem Tumorwachstum und zum Auftreten von Hirnmetastasen.
Die Zulassung von Ceritinib basiert auf den Ergebnissen von 2 offenen, 1-armigen Multicenterstudien (ASCEND-1 und -2). In der Phase-I-Studie ASCEND- ${ }^{2}$ wurden insgesamt 246 ALK-positive NSCLC-Patienten, die entweder mit Crizotinib vorbehandelt waren oder nicht, mit 750 mg Ceritinib therapiert, so Prof. Jürgen Wolf, Köln, auf der ZulassungsPressekonferenz ${ }^{3}$. In dieser Studie gab es auch eine Dosiseskalations- und Extensionsphase bis zur empfohlenen Dosierung von $750 \mathrm{mg}$. Vorbehandelte Patienten wiesen eine Gesamtansprechrate (ORR) von 56,4\%, eine mediane Dauer des Ansprechens von 8,3 Monaten und ein medianes progressionsfreies Überleben von knapp 7 Monaten auf. In ASCEND- $2^{4}$ wurden 140 mit Chemotherapie und Crizotinib vorbehandelte Patienten mit 750 mg Ceritinib weiterbehandelt. Diese Patienten erreichten eine ORR von 38,6\%. Das mediane Ansprechen dauerte 9,7 Mo- nate und das progressionsfreie Überleben 5,7 Monate.

Das Sicherheitsprofil von Ceritinib war allgemein konsistent in beiden Studien, wobei es keine neuen oder für die Substanzklasse unerwarteten unerwünschte Ereignisse gab. Die häufigsten unerwünschten Ereignisse betrafen gastrointestinale Nebenwirkungen, insbesondere Durchfallerkrankungen und Leberparameterveränderungen. Sie seien gut beherrschbar, verlangten allerdings, dass der Patient gut aufgeklärt und beraten werde, erklärte Prof. Michael Thomas, Heidelberg, auf einer Presseveranstaltung. Möglich sind dabei auch Therapiepausen oder eine Dosisreduktion um die Nebenwirkungen zu unterbinden.

\section{Richard Kessing, Zeiskam}

\footnotetext{
${ }^{1}$ Zykadia $^{\circledR}$, Novartis Pharma GmbH, Nürnberg

2 Kim DW et al. J Clin Oncol 2014; 32 (Suppl): abstr 8003

${ }^{3}$ Pressekonferenz: „Zykadia ${ }^{\circledR}$ - die neue Therapieoption für vorbehandelte ALK-positive Patienten mit nicht-kleinzelligem Bronchialkarzinom (NSCLC)“, Frankfurt, Juli 2015, Veranstalter: Novartis Pharma GmbH

${ }^{4}$ Mok T et al. J Clin Oncol 2015; 33 (suppl): abstr 8059
}

\section{Hohe Patientenzufriedenheit im Inhalationsalltag erzielen}

Obwohl für die Therapie obstruktiver Atemwegserkrankungen verschiedene Inhalationssysteme zur Verfügung stehen, sind viele Patienten weiterhin symp tomatisch. Das liegt u.a. an Anwendungsfehlern bei der Inhalation.

Dem Grundsatz der möglichst einfachen Bedienung folgt ein Inhalator ${ }^{1}$ mit der bewährten Fixkombination aus Budesonid und Formoterol. Er zeichnet sich durch eine intuitive Handhabung nach dem Prinzip „Öffnen - Inhalieren - Schließen“ aus. Um die Patientenzufriedenheit mit diesem Inhalator unter Alltagsbedingungen zu untersuchen, wird derzeit eine offene, nicht interventionelle Phase-IV-Studie mit Asthma- und COPD-Patienten durchgeführt ${ }^{2}$. Es sollen etwa 4000 Patienten in ca. 400 Prüfzentren im Zeitraum zwischen Juli 2014 und März 2016 in diese Studie eingeschlossen werden. Der jeweilige Beobachtungszeitraum beträgt 12
Wochen, als Ergebnisparameter werden Inhalator-Anwendungsfehler, Krankheitskontrolle, Patientenzufriedenheit, klinischer Zustand (Clinical Global Impression, CGI) und das Auftreten unerwünschter Ereignisse erhoben.

\section{Adhärenz verbessert sich}

Im Rahmen des 56. DGP-Kongresses 2015 wurden vorläufige Daten einer Interimsanalyse auf Basis von 1105 Patienten vorgestellt (Asthma: 68\%, COPD: 32\%), die auf den Inhalator neu ein- bzw. umgestellt wurden. Etwa jeder 2. Patient $(50,2 \%)$ wurde vorher bereits mit einer ICS-/ LABA-Kombination behandelt, ca. $16 \%$ hatten vor Studienbeginn noch nie einen Inhalator angewendet. „Die wichtigsten Gründe für die Einstellung der Patienten auf diesen Inhalator waren v.a. der geringe Trainingsaufwand $(44,4 \%)$ und die schnelle Einsatzbereitschaft $(39,1 \%)$ so- wie die erwartete Verbesserung der Adhärenz (37,4\%)“, erklärte Prof. Gillissen, Kassel. Aus dem Patientenkollektiv haben 492 Patienten bereits den kompletten 12-wöchigen Beobachtungszeitraum inklusive aller durchzuführenden Visiten abgeschlossen (Eingangsuntersuchung in Woche 1 , Zwischenuntersuchung nach 4 Wochen, Abschlusserhebung nach 12 Wochen). Bei ihnen kann die Änderung des klinischen Zustands im Vergleich zur erstmaligen Verordnung des Inhalators bei Studienbeginn beurteilt werden (CGI): Bei $72 \%$ dieser Patienten konstatierten die Prüfärzte eine Verbesserung. Dass auch die Patientenakzeptanz hoch ist, zeigt die Weiterverwendungsrate: $88 \%$ der ehemaligen Studienteilnehmer nutzten den Inhalator auch nach Beendigung der Studie weiter für die Inhalationstherapie.

\section{Nach einer Pressemitteilung (Teva GmbH)}

${ }^{1}$ DuoResp ${ }^{\circledR}$ Spiromax ${ }^{\circledR}$, Teva GmbH, Ulm

${ }^{2}$ Gillissen A et al. Pneumologie 2015; 69: P320 